\title{
Rigorous Bounds on the Analytic $S$ Matrix
}

\author{
Andrea Guerrieri® and Amit Sever@ \\ School of Physics and Astronomy, Tel Aviv University, Ramat Aviv 69978, Israel
}

(Received 14 August 2021; accepted 8 November 2021; published 15 December 2021)

\begin{abstract}
We consider a dual $S$-matrix bootstrap approach in $d \geq 3$ space-time dimensions which relies solely on the rigorously proven analyticity, crossing, and unitarity properties of the scattering amplitudes. As a proof of principle, we provide rigorous upper and lower numerical bounds on the quartic coupling for the scattering of identical scalar particles in four dimensions.
\end{abstract}

DOI: 10.1103/PhysRevLett.127.251601

Introduction.-An optimization problem can be viewed from either of two perspectives called, respectively, Primal and Dual. In the revived nonperturbative $S$-matrix bootstrap program of $[1,2]$, the space of scattering amplitudes is carved out numerically by solving an optimization problem in its primal form: the target to optimize is a physical observable; the constraints are the physical principles of analyticity, crossing, and unitarity. This approach has already been used to bound several classes of amplitudes. For example, in relation to integrable systems in two dimensions [3-12], and in higher dimensions for the case of standard model physics [13-17] and quantum gravity theories [18]. At the same time, any bootstrap scheme in terms of the primal variables involves making an ansatz for the amplitude and some type of truncation: as a result, the bounds are not strictly rigorous.

Our main motivation for constructing a dual formulation stems from the weak duality principle. Suppose we maximize an observable $\mathcal{O}$ over some space $D$. Then for any value of the dual variables in the dual space $\bar{D}$, the dual function $\overline{\mathcal{O}}$ will always provide an upper bound on $\mathcal{O}$, independently of how hard the primal problem is:

$$
\mathcal{O} \leq\left(\mathcal{O}_{*} \equiv \max _{D} \mathcal{O}\right) \leq\left(\overline{\mathcal{O}}_{*} \equiv \min _{\bar{D}} \overline{\mathcal{O}}\right) \leq \overline{\mathcal{O}}
$$

This weak duality principle allows one to construct a bootstrap scheme for generating rigorous bounds on the $S$ matrix, thus placing it in a similar footing to the conformal bootstrap [19]. In this Letter, we formulate a dual $S$-matrix bootstrap problem in $d \geq 3$ for the scattering of identical scalar particles that can be efficiently solved using SDPB [20-22]. A closely related-albeit nonlinearformulation was pioneered long ago in a series of papers

Published by the American Physical Society under the terms of the Creative Commons Attribution 4.0 International license. Further distribution of this work must maintain attribution to the author(s) and the published article's title, journal citation, and DOI. Funded by SCOAP ${ }^{3}$.
[24-28] and used to put rigorous bounds on the $\pi^{0} \pi^{0}$ scattering amplitude in four dimensions. Moreover, an alternative dual formulation has been constructed recently using the Mandelstam representation [29].

Before illustrating our strategy, we shall review the logic behind the dual approach in a simpler example. (See Chap. 5 of Ref. [30] for a general introduction to dual problems.) We consider the following toy problem-see Fig. 1

$$
\mathcal{O}_{*} \equiv \max _{\{x, y\}}\left\{\mathcal{O}(x, y) \mid x^{2}+y^{2} \leq 1\right\},
$$

with the primal objective taken to be

$$
\mathcal{O}(x, y)=a x+b y .
$$

The variables $\{x, y\}$ are called primal variables. The starting point to derive the dual version of problem (2) is to write the Lagrangian function

$$
\mathcal{L}(x, y \mid \lambda) \equiv(a x+b y)+\lambda\left(1-x^{2}-y^{2}\right),
$$

introducing a dual variable for each constraint, as $\lambda \geq 0$. This Lagrangian satisfies the following identity

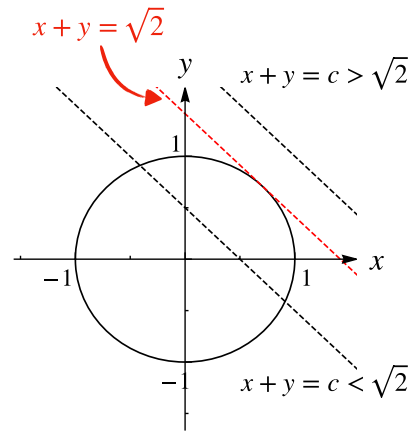

(a)

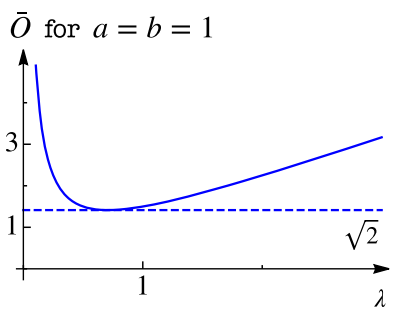

(b)
FIG. 1. (a) The geometric solution of problem $\max \left\{x+y \mid x^{2}+y^{2} \leq 1\right\}$. (b) The dual function $\overline{\mathcal{O}}(\lambda)=$ $\lambda+1 /(2 \lambda)$ of the same maximization problem. The minimum value is attained for $\lambda=1 / \sqrt{2}$ and coincides with the optimal value of the maximization problem. 


$$
\min _{\lambda}\{\mathcal{L} \mid \lambda \geq 0\}= \begin{cases}a x+b y & x^{2}+y^{2} \leq 1 \\ -\infty & \text { otherwise }\end{cases}
$$

Combining this with the max-min inequality yields

$$
\begin{aligned}
\mathcal{O}_{*} & \equiv \max _{\{x, y\}}\left\{\min _{\lambda}\{\mathcal{L} \mid \lambda \geq 0\}\right\} \\
& \leq \min _{\lambda}\{\overline{\mathcal{O}}(\lambda) \mid \lambda \geq 0\} \equiv \overline{\mathcal{O}}_{*},
\end{aligned}
$$

where the dual function is defined as

$$
\overline{\mathcal{O}}(\lambda) \equiv \max _{\{x, y\}} \mathcal{L}=\lambda+\left(a^{2}+b^{2}\right) /(4 \lambda) .
$$

The last inequality in Eq. (6) proves the weak duality principle anticipated in Eq. (1).

The dual objective in Eq. (7) is nonlinear, but it is concave [31]. For any fixed trial value of $\lambda \geq 0$ the dual function provides a rigorous upper bound for the quantity $a x+b y$. The difference between primal and dual objective is called duality gap and whether the duality gap is zero depends on the nature of the primal constraints. (See Refs. [30,32,33] for a set of sufficient conditions.)

In the following, we will apply this same logic to bound the quartic coupling between identical massive scalar particles in $d$ dimensions, showing explicit numerical results for $d=4$. We view our construction as a proof of principle that may be improved in the future.

The quartic coupling problem.-We list the set of constraints for the $2 \rightarrow 2$ scattering amplitude of identical scalars. First, the analyticity of the amplitude in the cut $s$ plane can be imposed using dispersion relations. The amplitude satisfies fixed- $t$ double subtracted dispersion relations for any real $-28<t<4$ in the $s$ plane with two cuts starting at $s=4$ and $u=4$, all expressed in units of the mass, [34-36]. Once combined with $s-u$ crossing, they can be put in the following form [36] (See Supplemental Material, Appendix A for the derivation [37].)

$$
\begin{aligned}
0 & =\mathcal{A}(s, t) \\
= & T(s, t)-T\left(s_{0}, t_{0}\right) \\
& -\frac{1}{\pi} \int_{4}^{\infty} d v\left[T_{v}(v, t) K\left(v, s, t ; t_{0}\right)+T_{v}\left(v, t_{0}\right) K\left(v, t, t_{0} ; s_{0}\right)\right],
\end{aligned}
$$

where $T_{v}(v, t)=\operatorname{Disc}_{v} T(v, t)$, and $\left(s_{0}, t_{0}\right)$ is an arbitrary subtraction point. Here,

$$
\begin{aligned}
K\left(v, s, t ; t_{0}\right)= & \frac{1}{v-s}+\frac{1}{v-4+s+t}-\frac{1}{v-t_{0}} \\
& -\frac{1}{v-4+t+t_{0}} .
\end{aligned}
$$

A function that satisfies the $\mathcal{A}$ constraint is automatically analytic in the cut $s$-plane and $s-u$ crossing symmetric, but not necessarily $s-t$ symmetric. Hence, this equation must be supplemented by the crossing constraint,
$T(s, t)=T(s, 4-s-t)$. This implies that the primal variables must consist of $s-t$ crossing symmetric functions, which in turn supports only even spins. (Instead of imposing crossing as a constraint, one may use the manifestly crossing symmetric dispersion relations of Refs. [38,39].)

Unitarity is most simply expressed as the probability conservation for fixed energy $s$ and $\operatorname{spin} \ell$

$$
1=\sum_{n}\left|S_{2 \rightarrow n}(s, \ell)\right|^{2} \geq\left|S_{2 \rightarrow 2}(s, \ell)\right|^{2},
$$

where $S_{2 \rightarrow n}(s, \ell)$ denotes the matrix element between twoparticle and $n$-particle states of spin $\ell$. The $S_{2 \rightarrow 2}$ amplitudes are given by

$$
S_{2 \rightarrow 2}(s, \ell)=1+i \rho^{2}(s) f_{\ell}(s),
$$

where $\rho^{2}(s)=\left[(s-4)^{[(d-3) / 2]} / \sqrt{s}\right.$ is the $d$-dimensional two-particle phase space factor and $f_{\ell}(s)$ the partial wave projection [40]

$$
f_{\ell}(s)=\frac{\mathcal{N}_{d}}{2} \int_{-1}^{1} d z\left(1-z^{2}\right)^{\frac{d-4}{2}} P_{\ell}^{(d)}(z) T[s, t(z)],
$$

with $t(z)=\frac{1}{2}(s-4)(z-1)$. For later convenience, we rephrase the unitarity constraint as the semidefinite (SDP) condition

$$
\mathcal{U}_{\ell}(s>4)=\left(\begin{array}{cc}
1-\frac{\rho^{2}}{2} \operatorname{Im} f_{\ell} & \rho \operatorname{Re} f_{\ell} \\
\rho \operatorname{Re} f_{\ell} & 2 \operatorname{Im} f_{\ell}
\end{array}\right) \geq 0 .
$$

The equivalence between Eqs. (10) and (13) can be seen by first noting that $\mathcal{U}_{\ell}$ is positive iff both its determinant and trace are positive. The positivity of the determinant is Eq. (10). The positivity of the trace follows from that of the determinant, as can be seen by noting that $\operatorname{det} \mathcal{U}_{\ell}=$ $2 \operatorname{Im} f_{\ell} \operatorname{tr} \mathcal{U}_{\ell}-4 \operatorname{Im} f_{\ell}^{2}-\rho^{2} \operatorname{Re} f_{\ell}^{2}$. Finally, in the elastic region of $4<s<16$, unitarity implies a stronger equality constraint

$$
\operatorname{det} \mathcal{U}_{\ell}(16>s>4)=0,
$$

instead of the inequality (13).

The quantity we want to bound is the quartic coupling, defined as the value of the amplitude at the crossing symmetric point [2]

$$
g_{0} \equiv \frac{1}{2 n_{0}^{(d)}} T\left(\frac{4}{3}, \frac{4}{3}\right) .
$$

Combining all the constraints, we write the Lagrangian (The sign \pm depends on whether we maximize or minimize $g_{0}$.)

$$
\begin{aligned}
\mathcal{L}^{ \pm}= & g_{0}+\iint_{\mathcal{D}} d s d t \mathcal{W}(s, t) \mathcal{A}(s, t) \\
& +\sum_{\ell \text { even }}\left[\int_{4}^{16} d s E_{\ell}(s) \operatorname{det} \mathcal{U}_{\ell}(s)\right. \\
& \left. \pm \int_{4}^{\infty} d \operatorname{str}\left[\boldsymbol{\Lambda}_{\ell}(s) \mathcal{U}_{\ell}(s)\right]\right]
\end{aligned}
$$


where $\mathcal{W}(s, t)$ and $E_{\ell}(s)$ are unconstrained dual variables imposing the analyticity (8) and elastic unitarity (14) in their validity domains, $\mathcal{D}$ and $s \in[4,16]$. Here, crossing has already been solved at the level of the primal variables by restricting to even spins only, $f_{2 n+1}(s)=0$. Finally, $\boldsymbol{\Lambda}_{\ell}(s)$ is a semidefinite positive matrix associated with the unitarity inequality constraint (13) that we impose for all energies. [A simple theorem states that the integrand $\operatorname{tr}\left[\boldsymbol{\Lambda}_{\ell}(s) \mathcal{U}_{\ell}(s)\right]$ is positive iff $\mathcal{U}_{\ell}(s) \geq 0$ for any $\boldsymbol{\Lambda}_{\ell}(s) \geq 0$.]

The dual variable space.-Here comes the important advantage of the dual formulation: omitting part of the constraints may weaker the bound, but due to the inequality $\min _{\lambda}\{\overline{\mathcal{O}}(\lambda)\} \leq \overline{\mathcal{O}}(0)$, it does not affect its rigor.

First, we simply set the dual variables associated with the elastic unitarity constraint (14) to zero, $E_{\ell}(s)=0$. This is because we do not know how to put it in SDP form. In the end, we find the elastic unitarity is satisfied in the region where the unitarity inequality is imposed;see also the numerical results [42].

Next, we discuss the dual function $\mathcal{W}(s, t)$. Its analyticity properties are connected to those of its associated constraint $\mathcal{A}(s, t)$ and its domain of validity. We consider a subspace of dual functions whose $s$-channel spin is bounded by a fixed integer $L$

$$
\mathcal{W}_{L}[s, t(z)]=\frac{\mathcal{N}_{d}}{2}\left(1-z^{2}\right)^{\frac{d-4}{2}} \sum_{\ell=0}^{L} w_{\ell}(s) P_{\ell}^{(d)}(z),
$$

where the sum runs over all spins, even and odd. Note that although we have solved the crossing constraint at the level of the primal variables by setting $f_{2 n+1}(s)=0$, the odd spins dual variables $w_{2 n+1}(s)$ impose nontrivial constraints. This is because the kernel in Eq. (8) is not $s-t$ symmetric.

At this point, we shall specify the domain $\mathcal{D}$ of $\mathcal{W}_{L}$ where we impose the $\mathcal{A}$ constraint. The larger this domain is, the more constraints we are imposing, hence the stronger the bound we get. The regime of validity of $\mathcal{A}$ is $-28<$ $t<4$ and any $s$. In addition, we demand the integral of $\mathcal{W}(s, t) T(s, t)$ in Eq. (16) to be diagonal in spin. This is achieved by letting $z$ run over the range $z \in[-1,1]$.

Combining the two conditions above implies that $0<s<32$. All in all, we take the integration domain to be $\mathcal{D}_{\mu}=\left\{(s, z) \mid z \in[-1,1] \wedge s \in\left[4, \mu^{2}\right]\right\}$, where $4<$ $\mu^{2}<32$ is a free parameter that we will tune later when solving the dual problem numerically. Later we will also comment about the possibility of enlarging further this integration domain-see the dual problem.

With these choices, the $\mathcal{A}$ term in the Lagrangian (16) takes the form

$$
\iint_{\mathcal{D}_{\mu}} d s d t \mathcal{W}_{L}(s, t) \mathcal{A}(s, t)=\sum_{\ell=0}^{L} \int_{4}^{\mu^{2}} d s w_{\ell}(s) a_{\ell}(s),
$$

where

$$
\begin{aligned}
a_{\ell}(s) \equiv & \frac{\mathcal{N}_{d}}{2} \int_{-1}^{1} d z\left(1-z^{2}\right)^{\frac{d-4}{2}} P_{\ell}^{(d)}(z) \mathcal{A}[s, t(z)] \\
= & \operatorname{Re} f_{\ell}(s)-\frac{\delta_{\ell, 0}}{n_{0}^{(d)}} T\left(s_{0}, t_{0}\right) \\
& -f_{4}^{\infty} d v \sum_{\text {Jeven }} k_{\ell, J}(v, s) \operatorname{Im} f_{J}(v),
\end{aligned}
$$

with the kernels $k_{\ell, J}$ given by

$$
\begin{aligned}
k_{\ell, J}(v, s) \equiv & \frac{\mathcal{N}_{d} n_{J}^{(d)}}{2 \pi} \int_{-1}^{1} d z\left(1-z^{2}\right)^{\frac{d-4}{2}} P_{\ell}^{(d)}(z) \\
& \times\left[P_{J}^{(d)}\left(1+\frac{2 t}{v-4}\right) K\left(v, s, t ; t_{0}\right)\right. \\
& \left.+P_{J}^{(d)}\left(1+\frac{2 t_{0}}{v-4}\right) K\left(v, t, t_{0} ; s_{0}\right)\right] .
\end{aligned}
$$

In Eq. (19) we used the fact that the imaginary part of the $\mathcal{A}$ constraint is automatically satisfied for real $s>4$. The even spin constraints $a_{2 n}(s)=0$ are also known as Roy equations [36]. They relate the real part of each partial amplitude to the absorptive parts that can be measured experimentally and they have been used in low energy QCD phenomenology; see Ref. [44] for a review. Notice also that the odd constraints $a_{2 n+1}(s)=0$ do not contain the odd spin real parts as we set $\operatorname{Re} f_{2 n+1}(s)=0$ by the choice of the primal variables.

The dual problem.-By plugging Eq. (18) into Eq. (16), and choosing conveniently the subtraction point at $s_{0}=t_{0}=4 / 3$, the Lagrangian becomes

$$
\begin{aligned}
\mathcal{L}^{ \pm}= & g_{0}\left(1-2 \int_{4}^{\mu^{2}} w_{0}(s) d s\right)+\sum_{\ell=0}^{L} \int_{4}^{\mu^{2}} d s w_{\ell}(s) \operatorname{Re} f_{\ell}(s) \\
& -\int_{4}^{\infty} d v \sum_{J \text { even }} \bar{w}_{J}(v) \operatorname{Im} f_{J}(v) \\
& \pm \int_{4}^{\infty} d v \sum_{J \text { even }} \operatorname{tr}\left[\boldsymbol{\Lambda}_{J}(v) \mathcal{U}_{J}(v)\right]
\end{aligned}
$$

where

$$
\bar{w}_{J}(v) \equiv \sum_{\ell=0}^{L}-\int_{4}^{\mu^{2}} d s w_{\ell}(s) k_{\ell, J}(v, s) .
$$

Before moving to the dual problem, we observe that using the symmetry $z \rightarrow-z$ of the even spin partial waves $f_{2 n}(s)$, we can extend the integration domain of the $w_{2 n}$ in the definition of Eq. (22) up to $\mu_{e}^{2} \leq 60$. This is achieved by integrating over half of the angles $z \in[0,1]$ in Eq. (19) and compensating by an overall factor of 2 . The region of integration for the odd spins is kept up to $\mu_{o}^{2} \leq 32$. In the next section we will find that having $w_{2 n}(s>12) \neq 0$ and $w_{2 n+1}(s>8) \neq 0$ is not feasible, so in practice one is forced to take $\mu_{e}^{2} \leq 12$ and $\mu_{o}^{2} \leq 8$. Yet, the $z \rightarrow-z$ 
symmetry is what allows the even spins bound to be larger than the odd one.

As in the toy problem (2), we define the dual functional by maximizing (minimizing) $\mathcal{L}^{+}\left(\mathcal{L}^{-}\right)$over the primal variables. The Lagrangian is linear in the primal variables and the extremization straightforward-see Supplemental Material, Appendix B for the details [37]. The final result is

$$
\min _{\substack{\boldsymbol{\Lambda}_{J}^{ \pm} \geq 0 \\ \pm \bar{w}_{J}(s) \geq 0}} \pm D^{ \pm} \equiv \min _{\substack{\boldsymbol{\Lambda}_{J}^{ \pm} \geq 0 \\ \pm \bar{w}_{J}(s) \geq 0}} \sum_{n=0}^{\lfloor L / 2\rfloor} \int_{4}^{\mu_{e}^{2}} d s X_{2 n}(s),
$$

with $J$ even. Here, $X_{J}=\left(\boldsymbol{\Lambda}_{J}\right)_{11}$, with $\boldsymbol{\Lambda}_{J}$ being the dual matrix that is associated with the unitarity constraint. It is given by

$$
\boldsymbol{\Lambda}_{J}^{ \pm}=\left(\begin{array}{cc}
X_{J} & \mp \frac{w_{J}}{2 \rho} \\
\mp \frac{w_{J}}{2 \rho} & \frac{1}{4}\left(\rho^{2} X_{J} \pm 2 \bar{w}_{J}\right)
\end{array}\right) \geq 0 .
$$

In Eq. (23) this constraint is imposed for $4<s<\mu_{e}^{2}$ and $0 \leq J \leq L$. In the complementary set, the following linear constraints are imposed

$$
\pm \bar{w}_{J \leq L}\left(s>\mu_{e}^{2}\right) \geq 0, \quad \pm \bar{w}_{J>L}(s>4) \geq 0 .
$$

Finally, the dual variable $w_{0}$ shall be normalized to

$$
\int_{4}^{\mu_{e}^{2}} d s w_{0}(s)=\frac{1}{2}
$$

Numerical results.-We have implemented the dual problem (23)-(26) in $d=4$ numerically. The summary of this investigation together with the one for the primal problem is plotted in Fig. 2. In red we depict the rigorously excluded region for $g_{0}$. In green, the allowed region obtained using primal numerics is indicated for comparison.

Primal.-We solve the primal problem by considering a manifestly crossing symmetric ansatz of the form

$T^{\text {primal }}(s, t, u)=\frac{c}{\rho_{s}-1}+\sum_{a+b \leq N_{\max }} c_{(a b)} \rho_{s}^{a} \rho_{t}^{b}+\mathrm{symm}$,

with $\rho_{x}=(\sqrt{8 / 3}-\sqrt{4-x}) /(\sqrt{8 / 3}+\sqrt{4-x})$ and by imposing unitarity numerically up to some spin $L_{\max }$ on a grid of points. For the maximum coupling case, convergence is remarkably fast. Conversely, the minimum coupling convergence is terrible: the different green lines in Fig. 2 correspond to increasing values of $N_{\max }$ indicating that primal convergence is far from being attained.

Dual.-The functional constraints (23)-(26) are implemented as follows. The dual variables $X_{2 n}$ and $w_{\ell}$ for $n=0, \ldots,\lfloor L / 2\rfloor$ and $\ell=0, \ldots, L$ are parametrized using a basis of functions, Chebyschev polynomials, for instance. We then choose a grid of points where we impose Eqs. (24) and (25); see Supplemental Material, Appendix G for details [37].

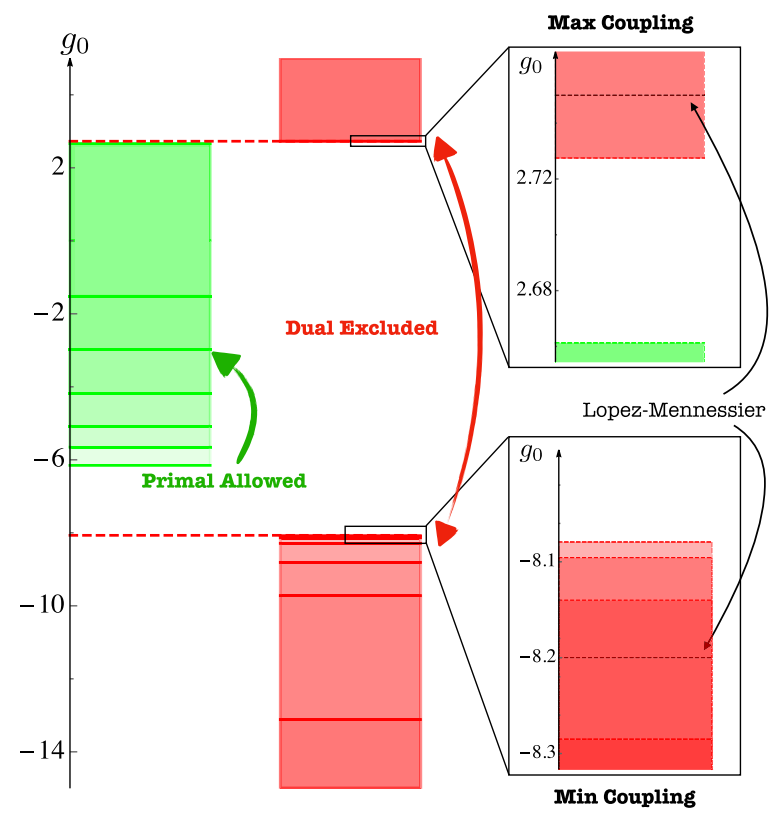

FIG. 2. Bounds on the quartic coupling $g_{0}$. On the left, we show in green the region determined by solving the primal problem, in red the one excluded by solving the dual (23). The bound on the maximum coupling converges fast both in the primal and dual problem and the gap is relatively small-right-top inset. The bound on the minimum coupling is hard to study using primal: the different green lines denote the numerical coupling for $N_{\text {max }}=5,8,11,14,17,20$. On the contrary, the red lines for $L=0, \ldots, 6$ show that dual convergence is achieved fasterright-bottom inset. In dashed black we report the best values of [28].

The set of constraints (25) on the sign of $\bar{w}_{J>L}$ is, however, infinite because the spin is unbounded. To implement them numerically, we first trivialize them at large $J$. This leads to a bound on the integration domain $\mu_{e}^{2} \leq 12, \mu_{o}^{2} \leq 8$ - see Supplemental Material, Appendix E for details [37]. We then introduce a spin cutoff $J_{\max }$ on the set (25) and gradually increase it. At intermediate spins larger than $J_{\max }$ we have also implemented the sign constraint near the two-particle threshold, see Supplemental Material, appendix F [37]. For the maximal coupling problem, we observe that beyond a certain value there are no dual constraint violations. For the minimal coupling problem, we always have some tiny violations at some $J>J_{\max }$, whose effect on the bound is negligible.

For the dual maximum coupling the simplicity of the primal mirrors into the dual. An almost optimal bound is attained using just $L=0$; adding further dual variables $w_{\ell}$ does not improve significantly the bound. For the minimum coupling, the dual numerical convergence is relatively slower as in the primal case. The red lines in Fig. 2 are obtained adding multipliers from $L=0$ up to $L=6$. We stress again that for any fixed $L$ the bounds obtained with large $J_{\max }$ convergence are rigorous. Increasing further $L$ will possibly make the gap smaller. (See Supplemental 

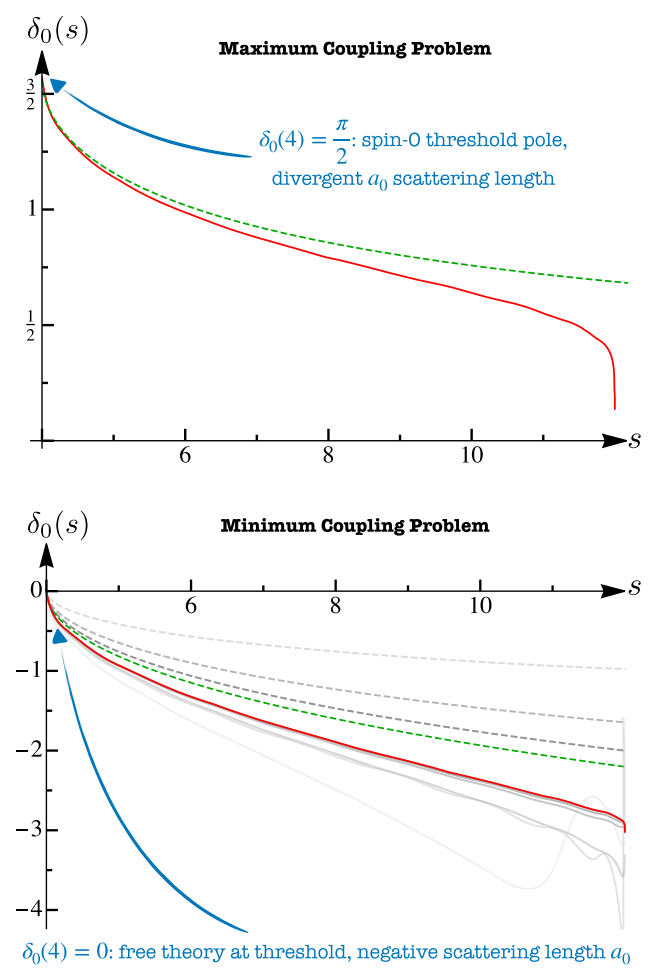

FIG. 3. The spin- 0 phase shifts, $\delta_{0}=(1 / 2 i) \log S_{0}$, as a function of the center of mass energy $s$ for the maximum (top) and minimum coupling (bottom). We plot in dashed-green and solid-red the phase shifts obtained respectively from the best primal and dual numerics. In the bottom figure, the dashed lines in grayscale correspond to increasing values of $N_{\max }$ up to $N_{\max }=20$ (in green); the solid lines in grayscale are obtained from the dual for different values of $L$, up to $L=6$ (in red). Although primal and dual results still differ, the physical content they describe is the same.

Material, Appendix $\mathrm{G}$ for a detailed analysis of the dual numerics, which includes Ref. [45].)

The different convergence rate of the two problems can be understood as follows. The quartic coupling $g_{0}$ can be measured using the dispersion relation $\mathcal{A}(4,0)$ in Eq. (8) subtracting at $s_{0}=t_{0}=4 / 3$

$$
\begin{aligned}
32 \pi g_{0}= & \operatorname{Re} T(4,0)-\frac{1}{\pi}-\int_{4}^{\infty} d v\left[T_{v}(v, 0) K(v, 4,0 ; 4 / 3)\right. \\
& \left.+T_{v}(v, 4 / 3) K(v, 0,4 / 3 ; 4 / 3)\right] .
\end{aligned}
$$

The integrand on the right-hand side is positive since $T_{v}\left(v, t_{0}\right) \geq 0$ for any $t_{0} \geq 0$. From Eq. (28) it is evident that maximizing the coupling is equivalent to minimizing the imaginary part (or the total cross section). On the other hand, when we minimize $g_{0}$ the optimal solution will have a total cross section that is as big as possible compatibly with unitarity. It is not surprising that primal convergence for the minimum coupling case is so hard since the imaginary part of our ansatz (27) does not grow at fixed $N_{\max }$.
In Fig. 3 we compare the primal and dual spin- 0 phase shift for the maximum and minimum coupling problem. The phase shifts tend to differ less as the duality gap shrinks. The threshold behaviors for primal and dual agree: the amplitude saturating the maximum coupling has a threshold singularity; the one saturating the minimum coupling a negative scattering length; see Supplemental Material, Appendix $\mathrm{H}$ for further details [37].

Discussion and outlook.-In this Letter, we proposed a dual approach to the $S$-matrix bootstrap based solely on the proven analyticity properties of scattering amplitudes. Our strategy consists of decomposing both crossing and unitarity into simpler constraints that can be systematically added to improve the bounds.

Still, there are some important questions to address. The first one concerns the duality gap. The dual problem we optimized numerically (23) is not the "mathematical dual" of the primal problem we solved following Ref. [6] and the duality gap does not necessarily close. In particular, in the dual, we imposed full unitarity up to $s=12$. This limitation follows from the dispersion relations we assumed in Eq. (8). (Several authors have tried to further extend the domain of validity of the Roy equations [39,46,47], see Refs. [48,49] for recent studies.) It might be interesting to use more refined dispersions [50] and check whether the gap can be further shrunk. In particular, it would be interesting to extend the applicability of the dual method beyond $s=16$, where nonelasticity is expected to kick in.

Although not proven, maximal analyticity is a typical working assumption made in bootstrap studies. It would be worth repeating our analysis under such a hypothesis and compare it with our rigorous bounds. It would also be interesting to extend the dual formulation to the case of massless particles. Clearly, in that case, the validity regime of our formulation shrinks to zero and some assumptions are needed in order to make progress. Related to that, it would be important to generalize the dual problem we formulated to bound Wilson coefficients in EFTs that recently have received a lot of attention [51-58].

Finally, one of the hardest challenges in the $S$-matrix bootstrap program concerns the inclusion of multiparticle processes. The proliferation of Mandelstam invariants is the bottleneck of manifestly crossing symmetric approaches. Single variable dispersion relations can overcome this issue. Whether the dual technology developed in this Letter can be used to tackle such challenging problems is an open question we think it will be important to address.

We thank M. Correia, J. Elias-Miró, A. Homrich, J. Penedones, A. Raclariu, P. Vieira, and A. Zhiboedov for useful discussion. We thank P. Vieira for comments on the draft. A. G. was supported by The Israel Science Foundation (Grant No. 2289/18). A. S. was supported by the Israel Science Foundation (Grant No. 1197/20). 
[1] M. F. Paulos, J. Penedones, J. Toledo, B. C. van Rees, and P. Vieira, The S-matrix bootstrap II: Two dimensional amplitudes, J. High Energy Phys. 11 (2017) 143.

[2] M. F. Paulos, J. Penedones, J. Toledo, B. C. van Rees, and P. Vieira, The S-matrix bootstrap. Part III: Higher dimensional amplitudes, J. High Energy Phys. 12 (2019) 040.

[3] N. Doroud and J. E. Miró, $S$-matrix bootstrap for resonances, J. High Energy Phys. 09 (2018) 052.

[4] Y. He, A. Irrgang, and M. Kruczenski, A note on the S-matrix bootstrap for the $2 \mathrm{~d} \mathrm{O}(\mathrm{N})$ bosonic model, J. High Energy Phys. 11 (2018) 093.

[5] L. Córdova and P. Vieira, Adding flavour to the S-matrix bootstrap, J. High Energy Phys. 12 (2018) 063.

[6] M. F. Paulos and Z. Zheng, Bounding scattering of charged particles in $1+1$ dimensions, J. High Energy Phys. 05 (2020) 145.

[7] A. Homrich, J. Penedones, J. Toledo, B. C. van Rees, and P. Vieira, The S-matrix bootstrap IV: Multiple amplitudes, J. High Energy Phys. 11 (2019) 076.

[8] J. E. Miró, A. L. Guerrieri, A. Hebbar, J. Penedones, and P. Vieira, Flux Tube S-matrix Bootstrap, Phys. Rev. Lett. 123, 221602 (2019).

[9] L. Córdova, Y. He, M. Kruczenski, and P. Vieira, The O(N) S-matrix monolith, J. High Energy Phys. 04 (2020) 142.

[10] C. Bercini, M. Fabri, A. Homrich, and P. Vieira, S-matrix bootstrap: Supersymmetry, $Z_{2}$, and $Z_{4}$ symmetry, Phys. Rev. D 101, 045022 (2020).

[11] A. L. Guerrieri, A. Homrich, and P. Vieira, Dual S-matrix bootstrap I: 2D theory, J. High Energy Phys. 11 (2020) 084.

[12] M. Kruczenski and H. Murali, The R-matrix bootstrap for the $2 \mathrm{~d} \mathrm{O}(\mathrm{N})$ bosonic model with a boundary, J. High Energy Phys. 04 (2021) 097.

[13] A. L. Guerrieri, J. Penedones, and P. Vieira, Bootstrapping QCD Using Pion Scattering Amplitudes, Phys. Rev. Lett. 122, 241604 (2019).

[14] A. Bose, P. Haldar, A. Sinha, P. Sinha, and S. S. Tiwari, Relative entropy in scattering and the S-matrix bootstrap, SciPost Phys. 9, 081 (2020).

[15] A. Guerrieri, J. Penedones, and P. Vieira, S-matrix bootstrap for effective field theories: Massless pions, J. High Energy Phys. 06 (2021) 088.

[16] A. Hebbar, D. Karateev, and J. Penedones, Spinning S-matrix bootstrap in 4d, arXiv:2011.11708.

[17] A. Bose, A. Sinha, and S. S. Tiwari, Selection rules for the S-Matrix bootstrap, SciPost Phys. 10, 122 (2021).

[18] A. Guerrieri, J. Penedones, and P. Vieira, Where Is String Theory?, Phys. Rev. Lett. 127, 081601 (2021).

[19] D. Poland, S. Rychkov, and A. Vichi, The conformal bootstrap: Theory, numerical techniques, and applications, Rev. Mod. Phys. 91, 015002 (2019).

[20] D. Simmons-Duffin, A semidefinite program solver for the conformal bootstrap, J. High Energy Phys. 06 (2015) 174.

[21] W. Landry and D. Simmons-Duffin, Scaling the semidefinite program solver SDPB, arXiv:1909.09745.

[22] The $2 \rightarrow 2$ scattering amplitude depends, in general, on two independent variables. In $d=2$, due to kinematical constraints, it is a function of one variable, which simplifies the problem. The $d=2$ dual bootstrap has been studied in Refs. [9,11,23].
[23] J. E. Miró and A. Guerrieri, Dual EFT bootstrap: QCD flux tubes, J. High Energy Phys. 10 (2021) 126.

[24] C. Lopez, A lower bound to the pi0 pi0 S-wave scattering length, Nucl. Phys. B88, 358 (1975).

[25] C. Lopez, Rigorous lower bounds for the pi pi p-wave scattering length, Lett. Nuovo Cimento 13, 69 (1975).

[26] C. Lopez and G. Mennessier, A new absolute bound on the pi0 pi0 S-wave scattering length, Phys. Lett. 58B, 437 (1975).

[27] B. Bonnier, C. Lopez, and G. Mennessier, Improved absolute bounds on the pi0 pi0 amplitude, Phys. Lett. 60B, 63 (1975).

[28] C. Lopez and G. Mennessier, Bounds on the pi0 pi0 amplitude, Nucl. Phys. B118, 426 (1977).

[29] Y. He and M. Kruczenski, S-matrix bootstrap in $3+1$ dimensions: Regularization and dual convex problem, J. High Energy Phys. 08 (2021) 125.

[30] S. Boyd and L. Vandenberghe, Convex Optimization (Cambridge University Press, Cambridge, England, 2004).

[31] This is always true for the dual problem: It follows from the fact that the Lagrangian is an affine function of the dual variables and that the pointwise supremum operation preserves convexity.

[32] D. Luenberger, Optimization by Vector Space Methods, 1st ed. (John Wiley and Sons, Inc, New York, 1997).

[33] D. Bertsekas, A. Nedic, and A. Ozdaglar, Convex Analysis and Optimization (MIT, Cambridge, MA, 2003).

[34] A. Martin, Extension of the axiomatic analyticity domain of scattering amplitudes by unitarity. 1., Nuovo Cimento A 42, 930 (1966).

[35] A. Martin, Extension of the axiomatic analyticity domain of scattering amplitudes by unitarity. 2, Nuovo Cimento A 44, 1219 (1966).

[36] S. M. Roy, Exact integral equation for pion pion scattering involving only physical region partial waves, Phys. Lett. 36B, 353 (1971).

[37] See Supplemental Material at http://link.aps.org/ supplemental/10.1103/PhysRevLett.127.251601 for a derivation of the fixed- $t$ double subtracted dispersion relations.

[38] G. Auberson and N. N. Khuri, Rigorous parametric dispersion representation with three-channel symmetry, Phys. Rev. D 6, 2953 (1972).

[39] G. Mahoux, S. M. Roy, and G. Wanders, Physical pion pion partial-wave equations based on three channel crossing symmetry, Nucl. Phys. B70, 297 (1974).

[40] We work in the normalization of Ref. [41] in which $P_{\ell}^{(d)}(z)={ }_{2} F_{1}[-\ell, \ell+d-3,(d-2) / 2,(1-z) / 2] \quad$ and $\mathcal{N}_{d}=\left\{(16 \pi)^{(2-d) / 2} / \Gamma[(d-2) / 2]\right\}$. In this normalization, the orthogonality of the partial waves takes the form $\left(\mathcal{N}_{d} / 2\right) \int_{-1}^{1} d z\left(1-z^{2}\right)^{[(d-4) / 2]} P_{\ell}^{(d)}(z) P_{\tilde{\ell}}^{(d)}(z)=\delta_{\ell \tilde{\ell}} / n_{\ell}^{(d)}$, with $\quad n_{\ell}^{(d)}=\left\{\left[(4 \pi)^{(d / 2)}(d+2 \ell-3) \Gamma(d+\ell-3)\right] /\right.$ $(\pi \Gamma[(d-2) / 2] \Gamma(\ell+1))\}$.

[41] M. Correia, A. Sever, and A. Zhiboedov, An analytical toolkit for the S-matrix bootstrap, J. High Energy Phys. 03 (2021) 013.

[42] It can be shown on general grounds that even with $E_{\ell}(s)=0$, the Lagrangian (16) leads to elastic functions, see Ref. [28] and the Supplemental Material, Appendix D [37]. This is not in contradiction with the Aks theorem [43], 
because the $S$-matrix constraints are not imposed to an arbitrary large $s$.

[43] S. O. Aks, Proof that scattering implies production in quantum field theory, J. Math. Phys. (N.Y.) 6, 516 (1965).

[44] G. Colangelo, J. Gasser, and H. Leutwyler, $\pi \pi$ scattering, Nucl. Phys. B603, 125 (2001).

[45] F. J. Yndurain, Rigorous constraints, bounds, and relations for scattering amplitudes, Rev. Mod. Phys. 44, 645 (1972).

[46] G. Auberson and L. Epele, A tool for extending the analyticity domain of partial wave amplitudes and the validity of roy-type equations, Nuovo Cimento A 25, 453 (1975).

[47] G. Auberson and S. Ciulli, A set of integral equations for pion pion scattering valid at all energies, Nuovo Cimento A 44, 549 (1978).

[48] A. Sinha and A. Zahed, Crossing Symmetric Dispersion Relations in QFTs, Phys. Rev. Lett. 126, 181601 (2021).

[49] R. Gopakumar, A. Sinha, and A. Zahed, Crossing Symmetric Dispersion Relations for Mellin Amplitudes, Phys. Rev. Lett. 126, 211602 (2021).

[50] A. L. Guerrieri and A. Sever (to be published).
[51] N. Arkani-Hamed, T.-C. Huang, and Y.-T. Huang, The EFThedron, J. High Energy Phys. 05 (2021) 259.

[52] M. B. Green and C. Wen, Superstring amplitudes, unitarily, and Hankel determinants of multiple zeta values, J. High Energy Phys. 11 (2019) 079.

[53] B. Bellazzini, J. E. Miró, R. Rattazzi, M. Riembau, and F. Riva, Positive moments for scattering amplitudes, Phys. Rev. D 104, 036006 (2021).

[54] A. J. Tolley, Z.-Y. Wang, and S.-Y. Zhou, New positivity bounds from full crossing symmetry, J. High Energy Phys. 05 (2021) 255.

[55] S. Caron-Huot and V. V. Duong, Extremal effective field theories, J. High Energy Phys. 05 (2021) 280.

[56] S. Caron-Huot, D. Mazac, L. Rastelli, and D. SimmonsDuffin, Sharp boundaries for the swampland, J. High Energy Phys. 07 (2021) 110.

[57] Z. Bern, D. Kosmopoulos, and A. Zhiboedov, Gravitational effective field theory Islands, low-spin dominance, and the four-graviton amplitude, J. Phys. A 54, 344002 (2021).

[58] S. Kundu, Swampland conditions for higher derivative couplings from CFT, arXiv:2104.11238. 